\title{
La diagonale du fou
}

Danielle Bonneau

\section{Q OpenEdition \\ Journals}

Édition électronique

URL : http://journals.openedition.org/shakespeare/1309

DOI : 10.4000/shakespeare.1309

ISSN : 2271-6424

\section{Éditeur}

Société Française Shakespeare

Édition imprimée

Date de publication : 1 novembre 1989

Pagination : 27-42

Référence électronique

Danielle Bonneau, «La diagonale du fou », Actes des congrès de la Société française Shakespeare [En ligne], 7 | 1989, mis en ligne le 01 janvier 2007, consulté le 06 mai 2019. URL : http:// journals.openedition.org/shakespeare/1309; DOI : 10.4000/shakespeare.1309 


\title{
Société Française Shakespeare
}

\author{
Actes des Congrès \\ 1985 - 1986 - 1987
}

\section{LA FOLIE}

\section{SHAKESPEARE ET LES ARTS \\ LE TRAGIQUE}




\section{LA DIAGONALE DU FOU}

Danielle BONNEAU

Le seul Fou des tragédies shakespeariennes est anonyme. En partie grâce à cet anonymat, sa substantifique moëlle s'enrichit des connotations du mot fool luimême - richesse abondamment explorée, dans une perspective d'évolution sociohistorique ou philosophique, par Barbara Swain ${ }^{1}$ et Enid Welsford ${ }^{2}$, et dans une perspective de synthèse linguistique par William Empson ${ }^{3}$. Nous allons donc ici anatomiser le Fou du Roi ; ceci nous amènera à poser le problème de ce que Victor Bourgy appelle sa portée morale, en d'autres termes, la stratégie du dramaturge pour exploiter et intégrer les potentialités du personnage au contexte dans lequel il se meut. Le titre un peu fou que j'ai choisi souligne à la fois cette stratégie et sa matérialisation a priori surprenante : Le Fou surgit au beau milieu de l'acte I et disparaît littéralement vers la fin de l'acte III, après une trajectoire météorique au cours de laquelle il aura non seulement suivi son maître, mais encore côtoyé tous les personnages importants, sauf Edmund et Cordelia. La métaphore ludique implicite dans la diagonale du Fou n'est cependant pas aussi gratuite qu'elle en a l'air : dans certains jeux de cartes, le joker n'a pas de valeur fixe mais peut, dans des circonstances particulières, entrer en jeu et se voir attribuer une valeur ${ }^{5}$; encore faudra-t-il définir cette valeur variable. Si le Fou est considéré en tant que pion, il apparâtra tantôt comme enjeu dans une partie que se livrent les protagonistes et par rapport à laquelle il aura une position marginale, voire un rôle d'observateur, tantôt comme mû de l'intérieur par des motivations qui le dépassent et transformé en force semi-autonome.

La première scène où il intervient nous semble capitale par ses allures de psychodrame. L'émergence soudaine du Fou, réclamé à trois reprises par un Lear décidément conditionné par ce chiffre, suscite ce qu'Empson appelle l'effet de mutualitét, variante de l'effet de miroir ; on entre de plain-pied dans l'interprétation 
érasmienne de la sagesse des fous, telle que la résume Victor Bourgy :

Il faut, prétend en effet l'auteur de L'Eloge de la Folie, reconnaître aux fous une qualité quin' est pas da dédaigner : "seulsils sont francs et véridiques" (section XXXVI). Mieux encore: ils peuvent l' être impunément, puisqu' ils sont fous et quel' on ne saurait leur tenir rigueur de leurs paroles. Ce qui vaudrait à un sage de perdre sa tête reste chez le fou un simple objet de divertissement et ce n'est pas là un simple avantage, car c'est finalement chez lui que la vérité a trouvé refuge, cette vérité qui est un don spécial des dieux aux simples d'esprit?.

Simple sans doute, fou "naturel" ce bouffon qui, d'entrée de jeu, propose à Kent et à Lear de leur passer son bonnet - le coxcomb - insigne de sa profession, mais aussi de la folie des sages, que Foly distribuait généreusement aux plus sots d'entre les sots à la fin de $A$ Satyre on the Three Estates de Sir David Lindsay'. Cette toute première intervention du Fou, geste autant que parole, polarisantl'attention sur le trait d'union visible et symbolique du bonnet, s'avère le point de départ d'une reconstruction par le sage fol de ce qui a $e u$ lieu de manière irréversible - reconstruction non pas matérielle et logique des événements, de cause à conséquence, mais métaphorique, à travers énigmes, proverbes, vers de mirliton et bribes de chansons parodiques. Le Fou résume à sa manière paradoxale le résultat du Love game de la scène I, s'adressant ensuite avec une affectueuse impertinence à ce nuncle qu'il feint de ne pas avoir encore aperçu :

Why, this fellow has banished two on's daughters, and did the third a blessing against his will (I, 4, 101-103, p. 82)'.

Assurément, la folie du Roi rejetant Cordelia et bannissant Kent a déjà été unanimement reconnue entre-temps, mais le Fou va plus loin encore : l'offre du bonnet au Roi fournit l'occasion de souligner, en un raccourci prophétique, son imprévoyance, son dénuement et sa dépendance vis-à-vis de ses filles :

Would I had two coxcombs and two daughters ! (...) If I gave them all my living, I'd keep my coxcombs myself. There's mine. Beg another of thy daughters $(\mathrm{I}, 4,107-108, \mathrm{p} .82)$. 
Le sage fol apparaît donc bien comme diseur de vérité et clairvoyant. Son attaque frontale se voit mal accueillie par Lear, qui le menace du fouet ${ }^{10}$, et il change de mode d'expression, glisse habilement du particulier au général, dans un mouvement de navette tissant des correspondances qui se révèlera caractéristique de son discours : puisque Lear en est encore à appeler fiel la vérité, le Fou se rabat sur une vérité plus terre-à-terre, pastichant dans une sorte de comptine $(I, 4,117-126, p .82)$ les codes de conduite médiévaux comme le Caton $^{11}$, qui prônaient une sage prudence dans le comportement quotidien comme préalable aux récompenses spirituelles.

L'incompréhension du Roi rappelle, jusque dans le mot-clé, son refus d'admettre la vérité de Cordelia : «Nothing can be made out of nothing » $(\mathrm{I}, 4,131$, p. 83). Le Fou persévère cependant dans son enseignement dialectique (teach est utilisé à deux reprises, vers 114 et 137). Sa première énigme, sur la différence entre bitter fool et sweet fool, se réfere de nouveau à la scène d'introduction; on est en effet frappé par la ressemblance avec le début d'une moralité comme Magnyficence de John Skelton (1515-1523), à ceci près que les abstractions flatteuses qui dupaient (made a fool of) le Roi, l'amenaient à se ridiculiesr (make a fool of himself), se sont incarnées en ses filles, et surtout que la folie du Roi s'est intériorisée, si bien qu'il ne peut échapper à sa responsabilité :

That lord that counselled thee

To give away thy land,

Come place him here by me ;

Do thou for him stand,

The sweet and bitter fool

Will presently appear :

The one in motley here,

The other found out-there». (I, 4, 138-145, p. 83).

En fait, le Fou propose à Lear de jouer un rôle dans une pantomime qu'il est loisible d'étoffer par l'imagination : sans avoir les motivations d'Hamlet, il souhaite lui aussi piéger la conscience du Roi, l'amener à s'identifier au bitter fool qui se prenait pour un sage. Grattez un roi, vous trouverez un fou, et de surcroît un fou de naissance :

LEAR. Dost thou call me fool, boy?

FOOL. All thy other titles thou hast given away ; that thou wast born with (I, 4, 146-148, p. 83).

Le Roi ne saurait se soustraire à la loi commune, il est embarqué dans la Nef des Fols de ce Monde de Sebastian Brandt (1497), ce monde dont il dira plus tard 
à Gloucester :

When we are born we cry that we are come

To this great stage of fools (IV, 6, 184-185, p. 157).

Si quiconque vient au monde naît coiffé du bonnet de folie, on comprend que tout le monde se mêle d'être fou et qu'il soit impossible d'obtenir un monopole $(\mathrm{I}, 4,149-153, \mathrm{p} .83)$ : le nombre des fous est infini. Infinies également les ressources rhétoriques de ce Fou qui sert à Lear, pêle-mêle, l'énigme de l'œuf et des deux couronnes - coquilles vides - puis, par association d'idées, le jeu de mots sur la couronne, emblème de pouvoir que le Roi porte sur sa tête chauve et qu'il a cru bon de partager en deux, perdant et la tête et la couronne :

Thou hadst little wit in thy bald crown when thou gavest thy golden one away $(\mathrm{I}, 4,160-161, \mathrm{p} .83)$.

Toutes vérités qu'il débite sous couvert d'une irresponsabilité adroitement revendiquée :

If I speak like myself in this, let him be whipped that first finds it so (I, 4, 161-162, p. 83).

Dès cette première scène, le Fou se présente aussi comme le révélateur (un autre touchstone) d'un monde que la folly de Lear a déséquilibré. Intuitivement, le Fou perçoit ce qui n'est déjà plus le royaume de Lear comme un néant dont surgit, tout armé, un monde à l'envers, monde contre nature et monde des "gens nouveaux"12. Le Roi lui-même participe à l'inversion des valeurs, traduite en langage de fol par l'image grotesque de l'adulte recevant la fessée puis, plus subtilement, par l'antithèse paradoxale de l'émotion exprimée et du sentiment qui l'inspire :

Thou madest thy daughters thy mothers; for when thou gavest them the rod and puttest down thine own breeches,

Then they for sudden joy did weep, And I for sorrow sung,

That such a king should play bo-peep And go the fools among (I, 4, 168-174, p. 84).

Le dialogue suggère au passage que ce bouffon, sans doute gentle comme 
Will Somers, chante surtout lorsqu'il est triste ; sa gaîté mélancolique ${ }^{13}$ renvoie au schéma d'inversion qui se dessine, de même que son souhait d'avoir un précepteur de mensonge

Il est clair en outre que l'attitude des autres vis-à-vis du Fou permet de discerner de quel bord ils sont : il a valeur d'enjeu dans ce qui pourrait bien être une partie d'échecs. Les paroles de Goneril au début de I, 3 établissent le lien fondamental entre le Roi et le Fou, la liberté du Fou et l'autorité du Roi, lien que le Roi, obscurément, proclame en défendant le Fou, lien d'identification que Goneril reconnaît et qui la met en fureur. Dramatiquement, le Fou sera donc la cause immédiate de l'affrontement entre père et fille, recherché de propos délibéré par Goneril :

Did my father strike my gentleman for chiding of his Fool? Idle old man,

That still would manage those authorities

That he hath given away! Now, by my life,

Old fools are babes again, and must be used

With checks, as flatteries, when they are seen abused

(I, 3, 1-2, 17-21, pp. 77-78)

Si la colère froide de Goneril prend pour bouc émissaire «this your alllicensed fool» $(\mathrm{I}, 4,196, \mathrm{p} .85)$, en l'associant aussitôt à «other of your insolent retinue» (vers 197), c'est bien qu'il n'est pas simple prétexte à querelle, mais symbole au même titre que les chevaliers.

Au cours de cette première scène, les thèmes de l'inversion et du néant, du rien, se retrouvent étroitement imbriqués dans le discours du Fou : superficiellement, l'impression produite sera l'incohérence normale chez un fol; or cette incohérence apparente recouvre une cohérence profonde. Progressiveemnt, le Fou passe du «Tu n'as plus rien» (l'œuf vide, la couronne cassée en deux morceaux) au «Tu n'es plus rien», impliquant toujours que Lear en est responsable. Dans les paroles du Fou, de plus en plus graves, le rien de I, 1, résonne comme un écho angoissant, malgré, ou à cause de la bouffonnerie des comparaisons :

I had rather be any kind $o$ ' thing than a fool. And yet I would not be thee, nuncle. Thou hast pared thy wit o'both sides and left nothing $i$ ' the middle. Here comes one o' the parings. (...) Now thou art an $O$ without a figure. I am better than thou art now ; I am a fool ; thou art nothing. (...) That's a shelled peascod. (1, 4, 181-184, 188-190, 195, pp. 84-85).

L'écho du rien affecte également Goneril, qui au départ ne dit rien, par 
contraste avec «That glib and oily art/ To speak and purpose not» (I, 1, 224-225, $\mathrm{p}$. 69), mis à jour lorsqu'elle parle enfin, pour se contredire. Ses reproches concrétisent l'inversion du rapport d'autorité et la menace à peine voilée que cela représente pour Lear. Dans la bouche du Fou, qui dès lors ne participe plus réellement au dialogue et dont la fonction chorique devient primordiale, cette menace prend tout son sens à travers une évocation de sauvagerie bucolique : 1 'image du coucou dévorant la tête de son père adoptif $(1,4,211-212$, p. 85) annonce le sort de Gloucester, simulacre de Lear, aux yeux crevés par les hôtes qu'il a généreusement accueillis. L'allusion mystérieuseà la lumière qui s'éteint, plongeant le monde dans l'obscurité (vers 213), s'applique elle aussi à Lear et à Gloucester. Lear, comme Richard II, n'est plus que «Lear's shadow ${ }^{14}(. .$.$) Which they will make an obedient father» (vers 227$ et 231). Cet univers crépusculaire's est bien un monde tragique : la subversion de l'autorité légitime y est envisagée, non comme un défoulement passager, mais comme une véritable prise de pouvoir projetée parEdmund dans lascène 2 de l'acte I- 》Edmund the base / Shall top the le gitimate» (I, 2,20-21,p. 72) -etbut de lastratégie, identique à celle des sœurs, qu'il attribue à Edgar ; ainsi, le vieux père et ses biens se trouvent placés sous la tutelle de l'enfant (I, 2,71-74, p. 74), ce qui constitue une distorsion inquiétante du verset du Magnificat chanté au début de laFête des Fous : «ll a abattu les puissants et exalté les humbles» ${ }^{16}$.

Réduit au silence par la violence du conflit auquel il assiste, le Fou se retrouve seul, face à l'animosité de Goneril qui le chasse, après son maitre : «you, sir, more knave than fool, after your master $! \rtimes(\mathrm{I}, 4,311, \mathrm{p} .88)$. Il va changer de cible critique, passant de la satire de folly à la condamnation de knavery. Ironiquement, c'est Goneril elle-même qui fournit cette nouvelle antithèse entre fool et knave ${ }^{17}$. Le Fou se venge de l'insulte en classant aussitôt Goneril dans la catégorie knave - l'ingratitude et l'hypocrisie débusquées :

\author{
A fox, when one has caught her, \\ And such a daughter \\ Should sure to the slaughter, \\ If my cap would buy a halter (I, 4, 314-318, p. 89).
}

Il poursuivra cette attaque à la scène 5 en prophétisant l'accueil de Regan, "as like this as a crab' slike an apple» (I, 5, 15,p.91). S'il reprend ensuite sa fonction d'amuseur, de jester, et accumule les énigmes insolites, les paradoxes, la fatrasie traditionnelle du type, il s'y glisse cependant toujours des allusions voilées. Ainsi le calembour grivois sur lequel il fait une sortie désinvolte $(1,5,47-48, p .92)$ appartient au registre bouffon et vise à divertir les groundlings ; mais il annonce implicitement le thème de la sexualité qui va dominer les actes III et IV, réunissant cette fois les deux sœurs et Edmund. 
L'acte II voit surgir un nouvel avatar de la Folie, destiné à concurrencer le Fou de Cour : Edgar, qui n'est désormais plus rien (II, 3, 21, p. 104), après Lear, et se trouve en danger de mort avant lui, décide de simuler le Bedlam Beggar, sorte de fou innoffensif qui sollicite la charité des villageois par ses haillons, ses pitreries et ses prières, et cherche à inspirer la pitié par les mortifications qu'il s'inflige.

Nous retrouvons le caquet amusant, voire cruel, du Fou devant le pilori. S'alignant sur le ton sarcastique de Lear - "Makest thou this shame thy pastime? ? (II, 4, 6, p. 104) - il se moque des jarretières de bois que l'aggressivité de Kent, «over-lusty at legs» (vers 9-10), lui a valu : ses prévisions sur l'accueil de la fille cadette se vérifient. De plus, en clairvoyant, il montre à son maitre le chemin vers un élargissement de la critique aux dimensions d'une société où le faible, le pauvre, 1'humble, sont rejetés impitoyablement, une Babylone dont la Fortune serait la grande prostituée (II, 4, 50-51, p. 106). L'articulation du général au particulier, c'està-dire au cas de Lear, se fait par le biais du jeu de mots sur dolours/dollars (vers 52), à propos des sceurs, qui vont le mettre à la porte à la fin de cette même scène et se conduisent plus tard en putains à l'égard d'Edmund. La partie que Lear compte livrer, partie dont l'enjeu n'est plus le Fou mais Kent, est donc, à en croire le Fou, perdue d'avance. Pour tenter de compenser les accès de souffrance qui submergent le Roi - et l'on aura compris que le Fou ne fait jamais autant le fou que quand tout va de mal en pis, il souligne avec la fable des anguilles, puis celle du foin beurré, les caractéristiques fondamentales du monde inversé que Lear doit affronter : sa cruauté (on veut cuire les anguilles vivantes et on leur tape sur le coxcomb quand elles redresssent la tête) et sa perversité hypocrite (beurrer le foin signifie non l'améliorer, «in pure kindness», mais le rendre immangeable) (II, 4, 117-121, p. 108). Regan et Goneril vont traiter leur père comme un pantin et lui prouver que, dans son propre intérêt, il n'a pas besoin d' un seul chevalier et serait beaucoup mieux servi par leurs gens.

Auparavant, en tête-à-tête avec Kent et l'unique gentilhomme qui s'est lancé aux côtés de Lear dans cette course-poursuite à travers la nuit, le Fou a livré son testament spirituel : jouant sur l'ambivalence et l'antinomie des mots knave et fool, il oppose sagesse mondaine et sagesse spirituelle; cette profession de foi, inversée par rapport à celle d'Edmund, constitue un véritable Eloge de la Folie, au sens où Erasme l'entend pour le chrétien :

The codes of conduct of the worldly and of the true Christian, of the true philosopher according to Plato, are totally opposed. (...) love is a madness, a being ravished away from oneself, beyond reason ${ }^{18}$. 
Alors que dans I, 4 et ici même, le Fou semblait condamner, au nom de la sagesse traditionnelle, l'attachement de Kentenvers un astre sur le déclin, la chanson qui d'habitude lui sert à illustrer sous une autre forme le même propos retourne comme un gant l'antithèse sagesse/folie, pour conclure sur un chiasme :

That sir which serves and seeks for gain, And follows but for form, Will pack when it begins to rain, And leave thee in the storm;

But I will tarry, the fool will stay, And let the wise man fly.

The knave turns fool that runs away;

The fool no knave, perdy. (II, 4, 74-81, p. 106-107)

Comme s'il avait pris le temps de formuler une réponse à Goneril et ce qu'elle représente, le Fou intègre les diverses conceptions de la folie, en confrontant celle du soi-disant sage aux yeux du monde qui n'est qu'un coquin, à la folle sagesse de celui qui se sait fou aux yeux du monde - ce que Guillaume de la Perrière, Tolosain, appelle dès 1553 la Morosophie ${ }^{19}$, citant comme références Platon, les philosophes stoïques, les prophètes et apôtres. Le bouffon exprime ainsi ce que Kent incarne déjà - d'où l'échange de politesse final entre les deux "fous" (II, 4, 82, 83, p. 107) - la nécessité profonde d'obéir à une Loi qui prime toutes celles du nouveau pouvoir établi, d'être fidèle et donc fou, de cette folly of love $e^{20}$ illustrée par Antigone, dont Gloucester et Cordelia vont à leur tour faire une règle impérieuse de vie et de mort ${ }^{21}$.

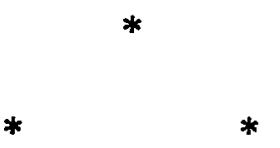

Seule à ne pas avoir perdu Lear dans la tempête de l'acte III, la figure en bonnet à clochettes et livrée bigarrée n'y a plus rien de l'observateur ironique ; trempé jusqu'aux os, il conseille à son maître d'accepter le monde à l'envers et de demander la bénédiction de ses filles. Car, avec le pessimisme de l'Ecclésiaste, il a brusquement une conscience aiguë de la fragilité humaine, et de la vanité de la sagesse : «Here's a night pities neigher wise men nor fools» (III, 2, 12-13, p. 118). C'est par sa simple présence que cette pitoyable incarnation de la faiblesse va susciter chez Lear son premier mouvement désintéressé : pour mettre à l'abri son Fou, il accepte de suivre Kent jusqu'à la hutte proposée : 
Come on, my boy. How dost my boy ? Art cold ? (...) Poor fool and knave, I have one part in my heart, That's sorry yet for thee» (III, 2, 68 et $72-73$, p. 120)

Après son cri du cœur, le Fou, toujours plein de conscience professionnelle, «labours to outjest (Lear's) heart-struck injuries» (III, 1, 16-17, p. 116). D'une certaine manière, il pave du même coup le chemin pour l'intervention d'Edgar, par des allusions obscènes qui associent les thèmes de l'imprévoyance et de la luxure : la Bible utilise folly comme synonyme de lewdness ou wantonness ${ }^{22}$. La braguette incontinente ne fait qu'aggraver son cas, et devient Poor Tom (III, 2, 27-30, p. 118). Assurément, la tradition veut que le Fou de Cour soit lui-même porté sur la chose ${ }^{23}$ et, d'ailleurs, n'arbore-t-il pas la braguette proéminente du fou tout court ? «Marry, here's grace and a cod-piece - that's a wise man and a fool» (II, 2, 40-41, p. 119), répondra-t-il à Kent. De même, sa prophétie parodique appartient au registre conventionnel : $s^{\prime}$ il s'agit, bien entendu, d'une satire du monde à l'envers qui s'est déjà instauré et a semé la «confusion» dans «la terre d'Albion», elle débouche cependant sur la vision d'une société utopique où tous les abus disparaîtraient, version idéalisée des méditations de Lear sur l'injustice. Brodant sur des vers que l'on croyait de Chaucer, l'orateur attribue ses prévisions à un futur Merlin (III, 2, 9596,p. 121), attribution suggestive à plus d'un titre : d'abord, elle place le Fou horstemps, tout en cadrant avec sa réputation de clairvoyant ; ensuite, selon diverses traditions sur la vie de Merlin ${ }^{24}$, il semblerait que, frappé de folie en châtiment d'un crime de sang, il aurait vécu en pleine nature à la manière d'un certain Poor Tom. Il appartiendrait donc à l'étrange confrérie des hommes des bois, ces green men que l'on retrouve dans les fêtes populaires médiévales héritées de rituels plus anciens, et qui, dotés du don de prophétie, étaient parfois enlevés de force par un roi auquel ils tenaient lieu de bouffon inspiré.

Ceci nous amène à ce que je nommerai la scène des transferts (III, 4). Le Fou y intervient très peu et, à l'exception de la comparaison entre le feu dans la nuit et «an old lecher' s heart» (III, 4, 108, p. 125), qui sert comme par hasard à introduire Gloucester, son discours tend de plus en plus vers la normale, jusqu'à se confondre pratiquement avec celui de Kent ou de Lear : «This cold night will turn us all to fools and madmen» (III, 4, 75-76, p. 124). Contrairement au Roi, fasciné par l'apparition de Poor Tom comme par un miroir et dont la raison s'abîme dans cette contemplation, le Fou ne voit dans le Bedlam que ce qu'il prétendêtre, une créature repoussante et terrifiante pour laquelle il ne semble guère éprouver de pitié. Il retrouve la transparence un peu puérile du simple d'esprit, de l'«innocent» (III, 6, 7, p. 129) : son rôle de critique de la folie humaine se transfere à Lear et à Poor Tom ; ce demier usurpe en fait une bonne partie de son registre, ce qui ne saurait surprendre de la part d'un simulateur : 


\begin{abstract}
It is upon the prototype of the natural fool that all the more sophisticated and artificial fools are based ${ }^{25}$.
\end{abstract}

L'aspect essentiellement incohérent de la fatrasie, feint et renforcé par Edgar pour suggérer le degré pathologique du dérangement mental, se retrouvera chez Lear à l'acte IV. Si l'idée fixe et l'hallucination caractérisent également Poor Tom (et Lear plus tard) parrapport au Fou de Cour, le Bedlam a un registre particulier de délire mystique, incantatoire à l'occasion (III, 4, 115-118, p. 126). Surtout, le niveau de la psychologie personnelle met en évidence, chez lui, des motivations semi-conscientes dans le choix du registre : le lien entre luxure et folie renvoie à la conception brandtienne du fou comme pécheur, et le prêche de Poor Tom fait clairement allusion aux péchés capitaux (III, 4,77-80, p. 124). Cette fixation sur la luxure prouve qu'Edgar, tout en assumant à sa façon la transgression de son père, source de leurs communs tourments ressent envers elle une révulsion puritaine qui ne se démentira jamais $(V, 3,167-171$, p. 176). La même obsession produira chez Lear fou des réactions plus ambiguës car délivrées, encore plus radicalement que celles du Fou, de toute censure psychologique et morale. Lorsque Poor Tom conclut la scène dans le langage mystérieux du clairvoyant, l'éclipse du sage fol ne peut que se constater :

Child Roland to the dark tower came $e^{26}$;

His word was still "Fie, foh and fum, I smell the blood of a British man (III, 4, 176-178, p. 128).

Reste l'étrange scène du procès (III, 6), qui voit s'achever la diagonale du Fou. Il se met au diapason de Poor Tom et entre dans le jeu, acceptant la fonction d'assesseur du Roi-Juge. Son dernier commentaire, «l'll go to bed at noon» (III, 6, 83, p. 132), épitomé du comportement paradoxal caractéristique du fol, évoque aussi, une ultime fois, le fait que tout désormais fonctionne à rebours - anomalie cristallisée dans la folie du Roi, pour lequel il ne peut plus grand-chose. Si Lear l'emmène apparemment avec lui, ce qui renvoie au sens équivoque du «Take the fool with thee» (I, 4, 312-313, p. 89), l'image qui nous reste du Fou aidant à porter le Roi jusqu'à la litière suggère comme une inversion positive du Diable des moralités emportant le Vice sur son dos. 
C'est peut-être, au fond, parce qu'il est demeuré anonyme que ce Fou, qui vient de sortir de la fable, rassemble en lui toutes les potentialité ${ }^{27}$ des conceptions philosophiques et morales de la Folie, de Brandt à Erasme : oxymore vivant, le fou sage est tout à la fois l'incarnation de la fragilité humaine, le fol, le bouffon amuseur, le porte-parole de la vision moralisatrice des codes de conduite, mais aussi l'avocat de la morosophie, folie douce sinon optimiste. D'autre part, selon les catégories $\mathrm{d}^{\prime} E c k h a r d t^{28}$, le comique de ce Fou s'avère essentiellement subjectif, c'est-à-dire qu'il fait rire aux dépens des autres. Il assure, de par sa profession, une fonction de comic relief, particulièrement intense dans les scènes graves (on songe au Portier de Macbeth).

Quant à sa trajectoire en diagonale, on peut tenter de l'expliquer en termes à la fois symboliques et dramatiques. Nous partirons d'une première constatation : le Fou intervient, comme c'est souvent le cas dans les moralités, à partir du moment où le protagoniste a déjà joué au fou en commettant une erreur fatale; le Fou intervient donc a posteriori, pour souligner l'inversion des valeurs qui résulte de cette erreur et annoncer les situations conflictuelles prévisibles. Dans la logique de la fable tragique, le Fou, sorte de Cassandre, parle mais n'influe pas sur les événements; il reste en marge comme seule voix critique autorisée, impuissante à modifier ce qui a été, mais mystérieusement annonciatrice de changements à venir.

Cette position privilégiée du Fou ressort de la comparaison avec Kent, qui n'est pas loin d'assumer la fonction du bouffon après le love game; c'est folie que de s'ériger en diseur de vérité sans la livrée protectrice ; puni par Lear, il récidive à 1'encontre d'Oswald, simulacre de Goneril, et se retrouve au pilori. En professionnel, le Fou prend le relais d'un Kent amateur dont il se moque, tout en appréciant ses bonnes intentions.

Après avoir constaté l'émergence d'un monde contre-nature où règne le calcul, rebaptisé sagesse, il témoigne à son tour qu'une nouvelle inversion est possible grâce à la sagesse des fous et cède littéralement la place, sortant du champ dramatique vidé de sa substance symbolique, laquelle a comme éclaté en fragments individuels. Le Roi-Fou engage un nouveau Fou de Cour, le simulateur Edgar-Poor Tom, son philosophe cynique ${ }^{29}$, à travers lequel il croit découvrir le degré zéro de la nature humaine, «the thing itself» (III, 4, 103, p. 125). Lear lui-même se métamorphose dès III, 6 en ce Lord of Mirsule ${ }^{30}$, Prince des Sots, qu'il était déjà pour ses filles à la fin de la scène 1 : symbole d'un contre-pouvoir anarchique et fantasque, il devait adopter, au cours de leurs affrontements, des attitudes bouffonnes qu'elles appelaient «pranks» (I, 4, 234, p. 86) ou «unsightly tricks» (II, 4, 152, p. 109). Ayant troqué sa couronne contre le bonnet de chasseur, s'étant vu offrir le bonnet du Fou, Lear se retrouve tête nue dans la tempête où s'établit le contact entre «his little world of man» (II, 1, 10, p. 116) et le macrocosme. Au cours de la scène du procès, forme traditionnelle de la bouffonnerie, la parodie de Trinité (Lear, le Fou, Poor Tom) recouvre une consubstantialité devenue manifeste dès l'acte IV : Lear, contaminé par le simulacre de folie, absorbe par surcroît les potentialités du Fou. L'oxymore incarné par ce dernier se voit transféré à la réalité dramatique : pour le Roi-Fou 
couronné à nouveau - de fleurs sauvages et d'herbes folles - et se confondant avec le green man, le passage obligé par «reason in madness» (IV, 6, 176, p. 157) comme, pour Gloucester, le paradoxe du fou guidant l'aveugle (IV, 1, 46, p. 140), permet d'essayer de remettre le monde à l'endroit, de recoller les morceaux de l'œuf du Fou. Si cette prouesse peut être tentée, c'est aussi parce que le Fou a passé le relais aux fools of love (Cordelia et Edgar), devenus tuteurs de leurs pères respectifs et, paradoxalement, neutralisant l'inversion de l'ordre naturel.

L'affinité profonde que l'on sent entre Cordelia et le Fou, l'ambiguïté verbale de «my pooor fool is hanged» $(\mathrm{V}, 3,303, \mathrm{p} .182)$, jointes à la sorte de frustration éprouvée devant la disparition prématurée du Fou, ont fait naître évidemment des hypothèses diverses : le Fou meurt, d'épuisement voire de chagrin, ou se suicide ; plus tentant, le Fou et Cordelia ne font qu'un, métaphoriquement et sans doute physiquement, par le truchement de l'acteur élisabéthain androgyne. Certes, on ne les voit jamais ensemble sur scène ; certes, ils détiennent tous deux la vérité, quoique celle du Fou apparaisse multiple, et comme diffusée par une transparence de medium.

Mais le refus d'accepter la dissolution du Fou dans la substance tragique repose avant tout sur un malentendu : le Fou de Lear n'est que le Fou du Roi, il n'a pas de passé et pas d'avenir. C'est un personnage sans personnage, c'est-à-dire sans histoire, à la fois conventionnel, complexe et énigmatique, dont les modes d'appréhension et d'action ne sont pas ceux de l'humanité ordinaire ; il est l'Etre par rapport au Paraître ou au Devenir. Il préside spirituellement aux minutes intemporelles de réconciliation, et je le verrais volontiers niché au creux de cette intemporalité, comme une sorte de génie du bien, jusqu'à ce que Prospero ait besoin d'Ariel. 


\section{NOTES}

1 Barbara Swain, Fools and Folly during the Middle Ages and the Renaissance, Columbia University Press (New York, 1932).

2 Enid Welsford, The Fool, his Social and Literary History, Faber (London, 1stpublished 1935, paperback edition 1968).

3 William Empson, The Structure of Complex Words, Chatto and Windus (London, 3rd edition 1977).

4 Victor Bourgy, Le Bouffon sur la Scène Anglaise au 16e siècle, OCDL, *Synthèses» (Paris, 1969). pp. 484-497.

5 William Willeford, The Clown, the Kingdom and the stage: A Study in the forms of our Relationship to folly, excerpt from his dissertation, Juris Druck + Verlag (Zurich, 1967), Introduction, p. 4.

6 Op.cit.. p. 108.

7 Op. cit. p. 488.

8 La première version est de 1540, la 2e de 1552 ; il n'est pas sans intérêt de noter, à la suite de Barbara Swain (op. cit., p. 165), que Lindsay est un des rares dramaturges anglais à refléter la tradition de la sottie, avec laquelle son séjour en France comme ambassadeur du roi d'Ecosse avait pu le familiariser.

9 Toutes les références sont celles de l'édition de G.K. Hunter, King Lear, New Penguin Shakespeare, 1972.

10 John Doran, A History of Court Fools, London, 1858, p. 291 : «Once engaged, the poor slave - for he was little else - could not sleep out of the palace unpermitted, without danger of a whipping when he returned. Neither could he lay aside his dress, without sanction of his master; and even then, were he to clap a sword on his thigh, and so try to pass abroad for a gentleman, and this offence came to the ears of the "king of the ribalds", the provost marshal of the King's house hold, the fool might reckon on being scourged till the blood ran down to his heelsw. La menace du fouet n'est donc pas vaine.

11 Barbara Swain, op. cit., pp. 13-16.

12 Swain, op. cit., pp. 99-100 : elle cite une sottie, la Farce nouvelle des gens nouveaulx qui mengent le monde et le logent de mal en pire. Les «gens nouveaux* se considèrent comme les maîtres du présent et les héritiers de la richesse du passé. Avec des matérieux de construction comme l'hypocrisie, la traitrise, la colère, ils édifient un monde nouveau qui s'écroule sur eux.

13 A cet égard, le Fou se révèle une sorte d'hybride, mi-Jaques, mi-Touchstone. Sa mélancolie semble n'avoir été qu'accentuée par le départ de Cordelia, puisque Lear s'étonne de cette profusion soudaine de chansons.

14 Au niveau del'espace théâtral concret, il me semble y voir comme une marque en creux, symbolique de cette perte de substance, du vide qu'est devenue la majesté de Lear : le 
lieu, vraisemblablement le château royal, où se déroule la scène d'abdication, devient lui-même non-lieu, shadow ; «I think our father will hence tonight», dit Goneril (I, 1, 284-285, p. 71); hence, en effet, mais sans retour possible vers cet ailleurs mythique où il était encore roi.

15 Toutes les scènes, depuis 1,2 jusqu'à la fin de l'acte III, se situent au crépuscule, pendant la nuit ou à l'aube.

16 Enid Welsford, op. cit., pp. 199-202 et Barbara Swain, op. cit., pp. $70-74$ : la Fête des Fous se célébrait dans l'église pendant les réjouissances de fin d'année, entre la SaintNicolas et la Chandeleur ; sa devise était les paroles du roi Salomon, Deposuit potentes de sede et exaltavit humiles.

17 Enid Welsford, op. cit., pp. 236-237:

The words "fool" and "knave" were constantly coupled together, but not always in quite the same way ;for sometimes they were treated as synonyms, sometimes emphasis was laid on the distinction between thems.

Pour Goneril, assurément, fool et knave sont tous deux péjoratifs, avec les sens distincts cependant d'imbécile et de coquin ; elle classe Lear et Albany dans la première catégorie, le Fou et la suite de cent chevaliers dans la seconde.

Corrwall, mais aussi Kent, utilisent knave comme insulte.

Lear est le seul à employer les deux termes comme synonymes dans un sens familier et affectueux, surtout en parlant du Fou (I, 4, 42, p. 80 et III, 2, 72, p. 120). Il englobe dans cette affection le Fou et Kent, qui sont respectivement umy pretty knaves (I, 4,96, p. 82) et umy friendly knave» (id., 94, p. 81).

La connotation péjorative de knave est réservée à Oswald, «my lord's knave» (id., 79, p. 81) : elle joue sur les deux sens du mot, «coquin» et «valet».

Il est intéressiant de signaler l'usage de fool et knave, combinant les sens péjoratifs et le double-entendre, dans Volpone V, 7, 89-91 (Ed. David Cook, Methuen University Paperback, London, 1962, p. 180), pièce jouée un an avant King Lear, en 1605, par la troupe de Shakespeare.

18 Barbara Swain, op. cit, p. 154, à propos de l'Eloge de la Folie. Elle cite également (pp. 36-37) les commentaires de Grégoire le Grand sur le Livre de Job, opposant la sagesse de ce monde à la sagesse des Justes : «this simplicity of the just is laughed to scorn, for worldly wise men believe the virtue of purity to be foolish.

19 Ce livre d'emblèmes didactique, publié à Lyon, est cité par Barbara Swain (op. cit., pp. 178-179). Prophètes et apôtres cautionnent l'idée qu'il n'est pas de «Prudence charnelle, ne Sagesse mondaine, qui ne soit folie envers Diews et les philosophes stoïques l'idée que celui qui se sait fou n'est pas loin d'être un sage (les propres paroles de Cicéron que Barbara Swain rapporte p. 12). Quant à Enid Welsford, elle cite la réprobation de Sénèque pour les bouffons et sa remarque sentencieuse : celui qui veut s'amuser d'un fou n'a pas à chercher loin, il lui suffit de se regarder (op. cit., p. 60).

20 J'emprunte cette formule à l'article de Charles Lyons, "The folly of Love», Revue des Langues Vivantes, Tijdschrift Voor Levende, XXXIV, p. 115.

21 Gloucester dit à Edmund : «IfI die for it, as no less is threatened me, the King my old master must be relieved» (III, 3, 16-17, p. 121), et plus tard à Lear : «My duty cannot suffer / T' obey in all your daughters' hard commands» (III, 4, 141-142, p. 126).

22 Wiliam Empson, Op. cit., p. 110 :

«Folly, the noun for the behaviour of a fool, gets an early implication "pleasure 
(probably sin)"; the N.E.D. gives 1303 as its first date for the sense «Lewdness», and Coverdale's version *because he hath committed folly in Israel* (Josh. VII, 15) was retained in the Authorised text though with ewickedness in the margin.»

C'est ce sens que l'on retrouve lorsqu'Othello tente de justifier le meurtre de Desdémone : «She turn'd to folly and she was a whore (V, 2, 135, William Skakespeare, The Complete Works, Ed. Peter Alexander, Collins, London, 1959, p. 1151).

23 William Empson, op. cit., p. 118, et William Willeford, op. cit., p. 11. Barbara Swain (op. cit., p. 65-66), parle de licence sexuelle.

24 Enid Welsford, op. cit., pp. 102-105. Je n'ai pu consulter l'article de Roland M. Smith, «King Lear and the Merlin Tradition» Modern Language Quarterly, VII, (1946), 153174.

La moralité de Lindsay, A Satire on the Three Estates, que nous avons déjà citée à propos de la première intervention du Fou, se termine, curieusement, par une version de la prophétie de Merlin en vers de mirliton latins (Barbara Swain, op. cit., p. 166).

25 Walter Kaiser, Praisers of Folly, Harvard university Press (Cambridge, Mass., 1963), p. 5.

Encore convient-il, pour apprécier la différenciation entre le Fou et le Bedlam, decerner dans le personnage de Poor Tom «le niveau de la psychologie feinte, qui ne sera autre (...) que celle du simple dans tout son illogisme et toute son imprécision, et le niveau de lapsychologie personnelle, constituée par les motifs réels et les sentiments profonds du simulateur à l' heure de son imitation. On devine sans peine que ce second élément était propre à enrichir de tout un jeu de nuances, comparable à une sorte de troisième dimension, le caractère de celui qui était assez subtil pour jouer d' une apparence de stupidité, (Victor Bourgy, op. cit., p. 203).

26 Cette dark tower du château de son père, maintenant annexé par le monde des knaves - celui de son frère et des filles de Lear - suggère en effet un danger mortel et une puissance maléfique (on songe au Lord of the Rings de Tolkien), tout en annonçant son futur rôle de David face à Goliath.

27 Shakespeare, malgré son small Latin and less Greek, connaissait peut-être la pièce latine de Nicholas Grimald, Archipropheta (1546-1547), où figure le seul fou complexe du thêâtre anglais avant celui de Lear, en la personne du bouffon d'Hérode, Gelasimus.

28 Eduard Eckhardt, Die lustige Person im älteren englischen Drama(bis 1642), Palestra, Hft 17 (Berlin, 1898).

29 John P. Cutts, «Lear's Learned Thebanw, Shakespeare Quarterly XIV, 4, 477-481. et E.M.M. Taylor, «Lear's Philosopher», Shakespeare Quarterly VI, 3, 364-365.

30 Il n'est pas sans intérêt de rappeler que la tragédie a été jouée d'abord à la Cour, le 26 décembre 1606, pendant cette période des fêtes de fin d'année héritées des Satumales ; d'ailleurs, jusqu'au règne d'Edouard VI, on nommait à la cour d'Angleterre (et à celle d'Ecosse) un Lord of Misrule, descendant du Prince de Noël ou Roi de la Fève de la tradition populaire, et spécialement chargé des divertissements. Cette tradition restait vivace dans les universités : à St John's College d'Oxford fut jouée, en décembre 1607, une pièce en latin, The Christmas Prince (Malone Society Reprints, 1922), où le Prince, face à la mort, est abandonné de tous sauf de son Fou. (Enid Welsford, op. cit., pp. 211213). 